\title{
Cardiovascular and Respiratory Response to Static Exercise in the Newborn Kitten
}

\author{
MARK D. PARRISH, JANEEN M. HILL, AND MARC P. KAUFMAN
}

University of California, Davis, California 95617

\begin{abstract}
Muscle contraction produces a reflex increase in blood pressure, heart rate, and minute ventilation in adults. To evaluate the role of this reflex in newborns, we compared the blood pressure, heart rate, and ventilatory responses to static contraction of the hindlimb muscles in sedated newborn and adult felines. The reflex response to muscle contraction was compared with the baroreflex, the chemoreceptor reflex, and the response to maximal stimulation of sciatic nerve afferents. With muscle contraction, newborn systolic blood pressure increased by $8.5 \pm 2.6 \%$, which was significantly less than the adult response of 15.9 $\pm 1.8 \%(p<0.025)$. Heart rate response to muscle contraction was less in newborns compared with adults, increasing by $1.4 \pm 0.5$ and $8.3 \pm 1.3 \%$, respectively $(p<$ $0.025)$. In contrast to heart-rate and blood-pressure responses, ventilatory responses to muscle contraction were similar in both age groups, increasing by $34 \pm 20$ and 34 $\pm 10 \%$ in newborns and adults, respectively. With stimulation of sciatic nerve afferents and with hypoxemia, blood pressure and heart rate increased similarly in both newborns and adults. When the baroreflex was elicited, heart rate decreased similarly in both age groups. We conclude that newborn cats have a reduced heart rate and blood pressure response to muscle contraction compared with the adult. We speculate that the postnatal development of this reflex is due to maturation of integrative and modulatory mechanisms in the CNS. (Pediatr Res 30: 95-99, 1991)
\end{abstract}

Blood pressure, heart rate, and minute ventilation increase reflexively during exercise (1). Chemically and mechanically sensitive unmyelinated $(\mathrm{C})$ and lightly myelinated $(\mathrm{A}-\delta)$ nerves form the afferent limb of this reflex arc (2). Brainstem autonomic centers integrate the afferent input and activate the efferent portion of the reflex arc $(3,4)$.

Although this reflex has been studied in adult mammals (5), including humans $(6,7)$, there is no information about the reflex in the immature animal. Clinical studies have shown that the hemodynamic response to exercise is more variable in children than in adults (8). Similarly, the ventilatory response at the onset of exercise is smaller in children for a given hypoxic stimulus (9). These findings suggest that there are important maturational changes in the reflex responses to exercise. We know nothing, however, about the reflex cardiovascular effects of muscle activity in neonates. It is possible that spontaneous muscle activity is an important factor regulating blood pressure in the newborn. However, teleologic thinking leads us to speculate that there is little need for a pressor reflex in immature animals when activity of

Received December 14, 1990; accepted March 18, 1991

Correspondence: Mark D. Parrish, M.D., Department of Pediatrics, University of California, Davis Medical Center, 2516 Stockton Blvd., Sacramento, CA 95817.

Supported by a grant from the California Affiliate of the American Heart Association, no. 90165. J.M.H. was supported by a fellowship from the American Heart Association, California Affiliate. large muscle groups is quite disorganized. Therefore, we hypothesized that the reflex response to static muscle contraction would be muted or absent in newborns. To explore this hypothesis, we compared the blood pressure, heart rate, and ventilatory responses to static contraction of the hindlimb muscles in newborns with those in adult felines. In addition, the reflex response to muscle contraction was compared with the baroreflex, the chemoreceptor reflex, and the reflex responses evoked by maximal stimulation of sciatic nerve afferents.

\section{MATERIALS AND METHODS}

Surgical preparation. Nine newborn (less than 1 wk of age) and eight adult cats (more than 6 mo of age) were sedated initially with ketamine, $25 \mathrm{mg} / \mathrm{kg}$, s.c. The right external jugular vein, carotid artery, and trachea were cannulated, and $\alpha$-chloralose, 25 to $50 \mathrm{mg} / \mathrm{kg}$, was given i.v. for additional anesthesia. The carotid line was attached to a Statham P23 pressure transducer (Statham, Cambridge, MA) and the tracheal cannula to a pneumotach for continuous recording of arterial pressure and minute ventilation, respectively. Heart rate was calculated beat to beat with a Gould Biotach (Gould, Inc., Oxnard, CA). Arterial blood gases were measured periodically (Radiometer ABL-3, Radiometer America, Inc., Westlake, $\mathrm{OH}$ ), and acid-base status was maintained in the normal range with i.v. sodium bicarbonite.

Cats were placed in a Kopf spinal unit (David Kopf Instruments, Tyunga, CA) to stabilize the hips and spine. The left calcaneal tendon was sectioned and attached to a force transducer. The right sciatic nerve was exposed and protected in a pool of $37^{\circ} \mathrm{C}$ mineral oil.

In half of the animals from each age group, a lumbosacral laminectomy was performed. After exposing the lumbosacral spinal cord and roots, the area was maintained in a $37^{\circ} \mathrm{C}$ mineral oil pool to prevent drying. Ventral roots $\mathrm{L} 7$ and S1 on the left side (muscle efferents) were sectioned, and the distal ends were placed on a stimulating electrode.

The animals without a laminectomy had the left sciatic nerve exposed. All visible branches of the nerve were cut except those supplying the triceps surae muscles. The nerve was placed on a stimulating electrode and protected by a pool of warm mineral oil.

Protocols. After a 30- to 60-min recovery period, during which heart rate, blood pressure, and minute ventilation stabilized, each animal underwent five experimental procedures. Twenty to 30 min elapsed between each procedure to permit the animal to restabilize.

First, we tested the cardiovascular and ventilatory responses to static muscular contraction as well as the reproducibility of these responses. Initially, and $20 \mathrm{~min}$ later, the triceps surae muscles were statically contracted for $60 \mathrm{~s}$ by electrically stimulating the left sciatic nerve or the L7-S1 ventral roots. The ventral roots were stimulated with current voltages two to three times motor threshold, a frequency of 30 to $40 \mathrm{~Hz}$, and a pulse duration of $0.1 \mathrm{~ms}$. The parameters for stimulating the left sciatic nerve 
( 1.5 to 2.5 times motor threshold, 30 to $40 \mathrm{~Hz}, 0.025 \mathrm{~ms}$ ) have been shown to be too weak to activate electrically group III and IV fibers $(10,11)$. Before stimulation, the triceps surae muscles were stretched to a resting tension from which maximal peak tension could be developed. Blood pressure, heart rate, and ventilation were monitored continuously for $60 \mathrm{~s}$ before, during, and after the contraction.

For the second protocol, we abolished afferent input from the contracting triceps surae muscle to the CNS by sectioning the L6, L7, and S1 left dorsal roots. For those animals in which contraction was produced by stimulating the left sciatic nerve, this nerve was sectioned proximal to the stimulating electrode. Contractions were evoked by using the aforementioned stimulation parameters.

In protocol 3, we assessed the cardiovascular and ventilatory responses evoked by high-intensity electrical stimulation of the cut end of the sciatic nerve. The right sciatic nerve was sectioned, and the proximal end attached to a stimulating electrode. After 1 min of baseline recording, the nerve was stimulated with a frequency of $20 \mathrm{~Hz}$, pulse duration of $0.75 \mathrm{~ms}$, and voltage of 7 $\mathrm{mA}$. This was at least 100 times the motor threshold and is known to activate both myelinated and unmyelinated fibers (12).

For protocol 4, we evaluated the cardiovascular effects of hypoxic stimulation of chemoreceptors. After $1 \mathrm{~min}$ of baseline measurements, taken while the animals were breathing room air, the animals were switched to a gas mixture of $95 \%$ nitrogen and $5 \%$ oxygen for $1 \mathrm{~min}$. Arterial blood gases were measured before and at the end of the hypoxic episode.

Finally, the effects of baroreceptor stimulation were assessed. Phenylephrine was infused rapidly into the jugular vein, and blood pressure, heart rate, and ventilation were recorded. Adults were given a dose of $10 \mu \mathrm{g} / \mathrm{kg}$. Because newborns are less responsive to this drug (13), a dose of $20 \mu \mathrm{g} / \mathrm{kg}$ was used for this age group.

Statistical analysis. During the intervention protocols, heart rate, blood pressure, and minute ventilation were recorded continuously for $60 \mathrm{~s}$ before each stimulus (baseline recording) and for another $60 \mathrm{~s}$ during each stimulus (stimulus recording). To summarize responses, peak heart rate and blood pressure were noted for each 60 -s period. Ventilatory volumes were integrated, and the total inspired volume during each 60 -s period was noted (minute ventilation). All values are reported as means \pm SEM. Between group and within group comparisons were performed using analysis of variance with a mixed design (one between and one within) (14). When significant differences were found, Tukey's test was used for post-hoc testing (15). A $p$ value of less than 0.05 was chosen as indicative of significant differences between means.

\section{RESULTS}

Baseline data. Table 1 contains descriptive information for the newborn kittens and adult cats. Also shown in Table 1 are control values for protocol 1 measurements of muscle tension, blood pressure, heart rate, and ventilation. Similar control measurements were obtained with each protocol. As noted in Table 1 , the adult body weight was approximately 25 times more than the newborn body weight, whereas the triceps surae muscles were over 80 times heavier in the adults than in newborns. When resting and developed muscle tension were divided by the weight of the muscle, newborns had significantly greater values. This suggests that newborn muscle performed significantly more work during the min of contraction than the adult muscle. However, because adult triceps were more hypertrophied than newborn muscle, we also show the muscle tensions as a percentage of body weight. Looked at this way, resting and developed tension were not significantly different between the age groups. Control resting and developed tensions were not significantly different between protocols 1 and 2 .

The stability of the preparation is documented by the lack of
Table 1. Baseline measurements

\begin{tabular}{lcc}
\hline & Newborn $(n=9)$ & Adult $(n=8)$ \\
\hline Age (d) & $1.6 \pm 0.4$ & $>6 \mathrm{mo}^{*}$ \\
Body wt (g) & $141 \pm 11$ & $3776 \pm 340^{*}$ \\
Triceps surae wt (g) & $0.43 \pm 0.04$ & $37.97 \pm 4.15^{*}$ \\
Triceps surae wt (\% body wt) & $0.305 \pm 0.012$ & $0.994 \pm 0.41^{*}$ \\
Resting tension (g/g triceps) & $46.5 \pm 5.6$ & $20.9 \pm 2.4 \dagger$ \\
Resting tension (g/g body wt) & $0.167 \pm 0.032$ & $0.204 \pm 0.015$ \\
Developed tension (g/g triceps) & $450 \pm 90$ & $137 \pm 16 \dagger$ \\
Developed tension (g/g body wt) & $1.39 \pm 0.30$ & $1.34 \pm 0.14$ \\
Systolic pressure (mm Hg) & $70 \pm 3$ & $169 \pm 7^{*}$ \\
Diastolic pressure (mm Hg) & $51 \pm 3$ & $133 \pm 5^{*}$ \\
Mean pressure (mm Hg) & $57 \pm 3$ & $145 \pm 6^{*}$ \\
Heart rate (bpm) & $262 \pm 21$ & $209 \pm 10 \dagger$ \\
Minute ventilation (mL/min) & $77 \pm 15$ & $709 \pm 115^{*}$ \\
\hline
\end{tabular}

$$
\begin{aligned}
& { }^{*} p<0.001 . \\
& \dagger p<0.05 .
\end{aligned}
$$

change in baseline measurements throughout the various protocols. For newborns, mean blood pressure was $57 \pm 3.0 \mathrm{~mm} \mathrm{Hg}$ at the beginning of the experiment (protocol 1) and $51 \pm 3.3$ $\mathrm{mm} \mathrm{Hg}$ at baseline in protocol $5(p=0.07)$. For the adults, mean blood pressure was $141 \pm 8$ and $144 \pm 6 \mathrm{~mm} \mathrm{Hg}$, respectively $(p=0.58)$. Newborn heart rate was $262 \pm 22$ for protocol 1 and $250 \pm 26$ for protocol $5(p=0.23)$. Adult heart rates were $206 \pm 11$ and $200 \pm 10(p=0.38)$. Newborn minute ventilation was $77 \pm 15$ and $79 \pm 17 \mathrm{~mL} / \mathrm{min}(p=0.84)$. Minute ventilation in the adults was $703 \pm 128$ and $593 \pm 133 \mathrm{~mL} / \mathrm{min}$ $(p=0.24)$.

Muscle contraction. Two muscle contractions were performed in each animal before severing the muscle afferents. Because the responses to these two muscle contractions were not significantly different, the results were pooled for the purposes of analysis. In response to muscle contraction, systolic blood pressure increased by $8.5 \pm 2.6 \%$ in newborns and $15.9 \pm 1.8 \%$ in adults. Although this was a significant change from baseline for both groups, the newborn increase was less than that of the adult group $(p<$ $0.025)$. After afferent input from the muscle was abolished (protocol 2), systolic blood pressure did not change significantly in the newborn group during muscle contraction $(1.6 \pm 1.8 \%)$ and increased only slightly in the adults $(3.7 \pm 1.7 \% ; p=$ not significant, newborns versus adults). For both groups, the bloodpressure response after severing muscle afferents was significantly less than the response with an intact reflex arc. Similar agedependent differences were found when mean blood pressure (mm Hg) was analyzed (Fig. 1A). Thus, newborn blood pressures changed very little during muscle contraction, and this was significantly different from the response seen in the adults.

Heart rate response to muscle contraction is summarized in Figure $1 B$, displayed as $\mathrm{bpm}$. Although heart rate increased slightly in the newborn $(1.4 \pm 0.5 \%, p<0.05)$, this was not significantly different from the response after abolition of muscle afferents $(1.0 \pm 1.1 \%)$. In the adult, heart rate increased by 8.3 $\pm 1.3 \%$ during contraction. This was significantly different from the newborn response $(p<0.025)$ and was different from the heart rate response after severing the muscle afferents $(3.0 \pm$ $2.0 \%, p<0.01)$.

Ventilatory responses to muscle contraction are summarized in Figure $1 C$. Both age groups showed significant increases in minute ventilation during muscle contraction, and these increases were abolished after severing muscle afferents. There was no differences between the age groups.

Sciatic stimulation. The response of systolic blood pressure to sciatic stimulation is summarized in Figure 2 and is compared with the systolic pressure changes with muscle contraction. Maximal sciatic nerve stimulation produced significantly greater increases in blood pressure and heart rate than did muscle contraction. However, unlike muscle contraction, there were no significant differences between newborns and adults during sciatic 

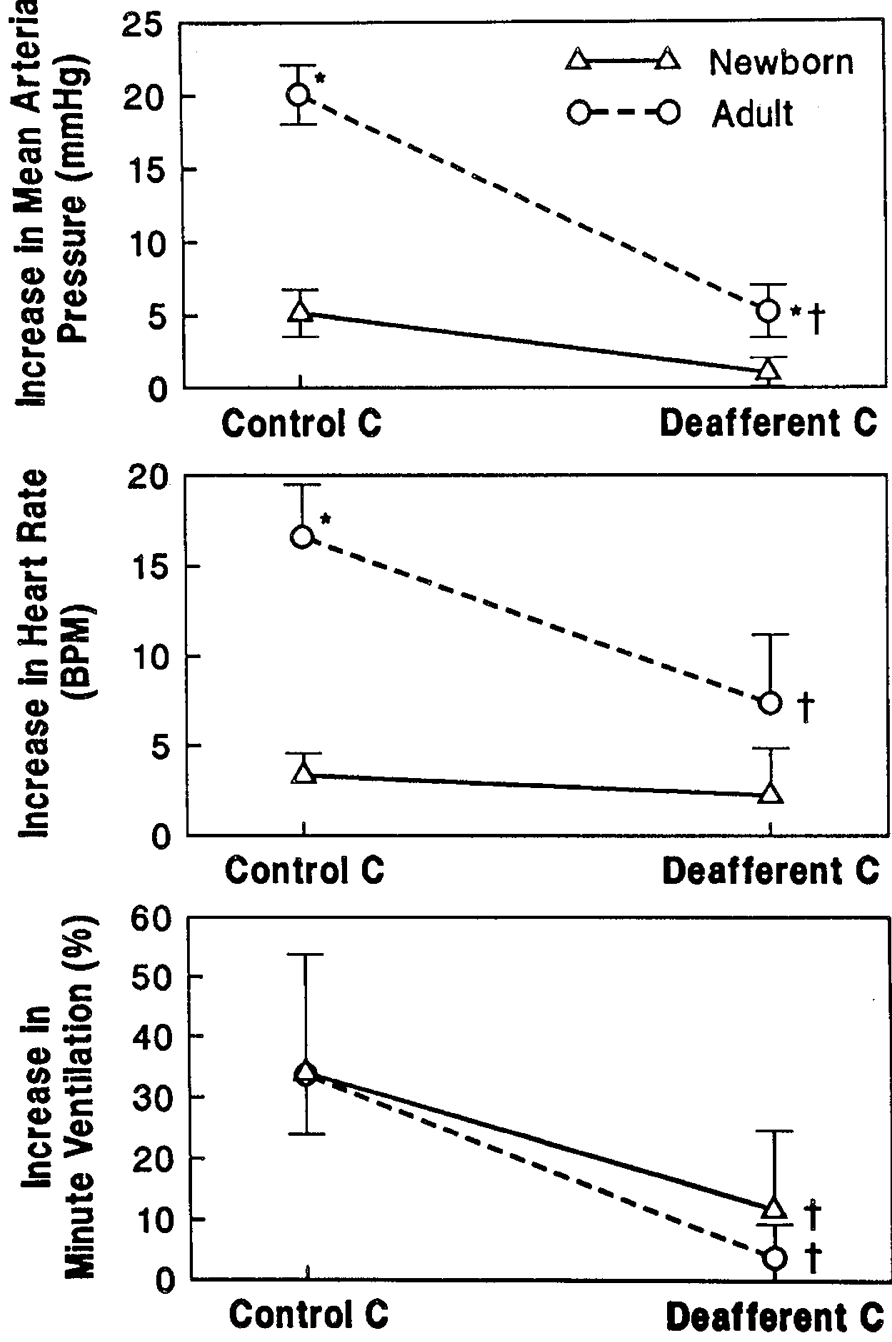

Fig. 1. Increases in blood pressure, heart rate, and minute ventilation are displayed for the control contraction (protocols 1 and 2) and for the contraction after muscle afferents were severed (protocol 3). Values are displayed as means \pm SEM. $C$, contraction; *, different from newborn group $(p<0.05)$; $\dagger$, different from control contraction $(p<0.05)$.

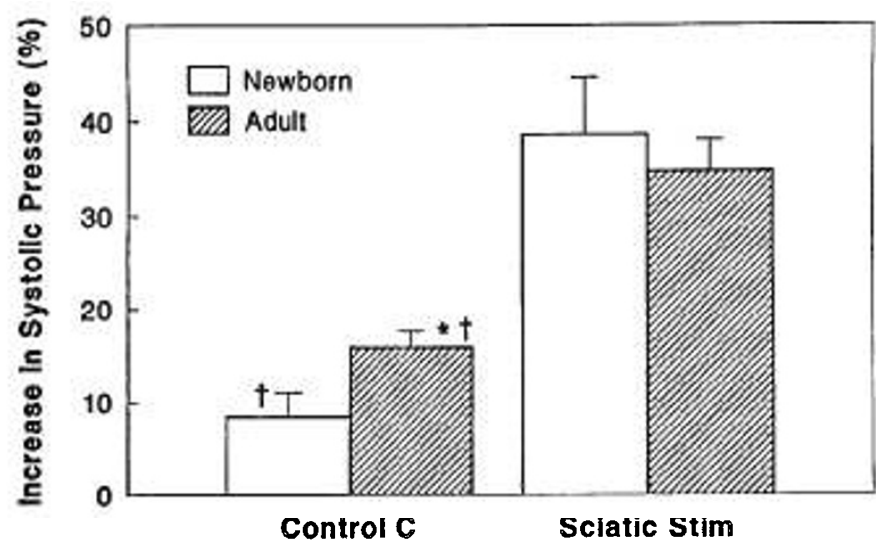

Fig. 2. Increase in systolic blood pressure as a percentage of change from the baseline value is shown for the control contraction (protocols 1 and 2) and for the maximal sciatic nerve stimulation (protocol 4). Values are displayed as means \pm SEM. $C$, contraction; Stim, stimulation; *, different from newborn group $(p<0.025)$; $\uparrow$, different from sciatic stimulation $(p<0.01)$. stimulation. Heart rate (not shown) increased by $9.5 \pm 3.9$ and $12.3 \pm 4.2 \%$ in newborns and adults, respectively $(p=\mathrm{NS}$, newborn versus adult). Minute ventilation increased by $47 \pm$ $21 \%$ in newborns and by $58 \pm 34 \%$ in adults ( $p=N S$, newborn versus adult).

Hypoxemia (chemoreceptor stimulation). Breathing $5 \%$ oxygen for $1 \mathrm{~min}$, both newborns and adults achieved similar levels of arterial hypoxemia with no acidosis $\left[\mathrm{PO}_{2}=22 \pm 2 \operatorname{torr}(2.9 \mathrm{kPa})\right.$ and $24 \pm 1$ torr $(3.2 \mathrm{kPa})$ in newborn and adult groups, respectively]. This hypoxemia produced significant increases in blood pressure in both age groups, and there was no significant difference between newborns and adults (Fig. 3). Although newborns appeared to have a decrease in heart rate with hypoxemia (Fig. 3 ), there was no statistically significant change from baseline and no difference between newborns and adults. As shown in Figure 4 , hypoxemia produced significant increases in minute ventilation in adults but not in newborns.

Baroreceptor stimulation. Phenylephrine injection produced similar increases in blood pressure in newborns and adults (Fig. 5 ). The $25 \%$ increase in mean blood pressure after phenylephrine infusion was similar to the increase in mean pressure produced by muscle contraction in the adult group in protocols 1 and 2 . The decrease in heart rate after phenylephrine infusion was significant for both groups $(-40 \pm 7 \%$ in newborns and $-55 \pm$ $6 \%$ in adults, $p=$ NS, newborns versus adults). Only the adult group achieved a significant decrease in minute ventilation compared with baseline values, although comparison of the percentage of change showed no significant difference between the two age groups (Fig. 5).

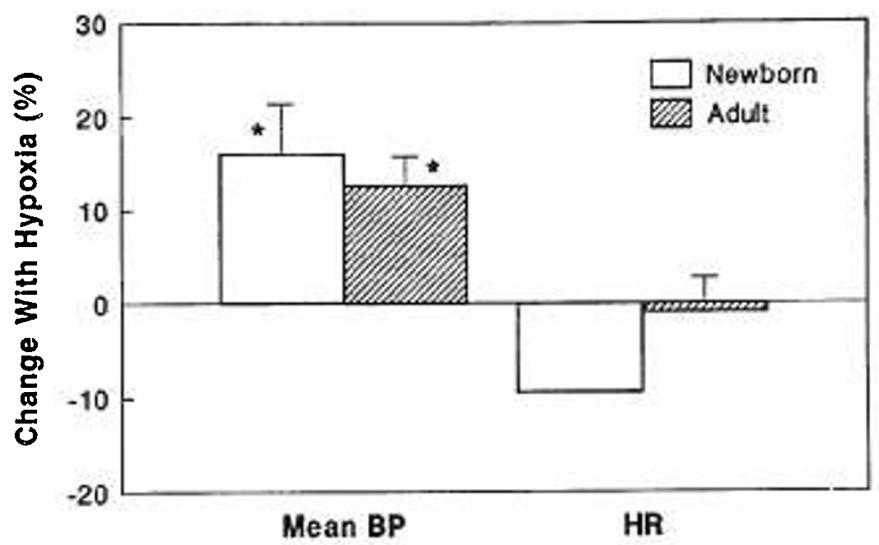

Fig. 3. Blood pressure and heart rate response to hypoxemia is shown as a percentage of change from the baseline value (protocol 5). Values are displayed as means \pm SEM. $B P$, blood pressure; $H R$, heart rate; *, different from the normoxemic baseline $(p<0.05)$.

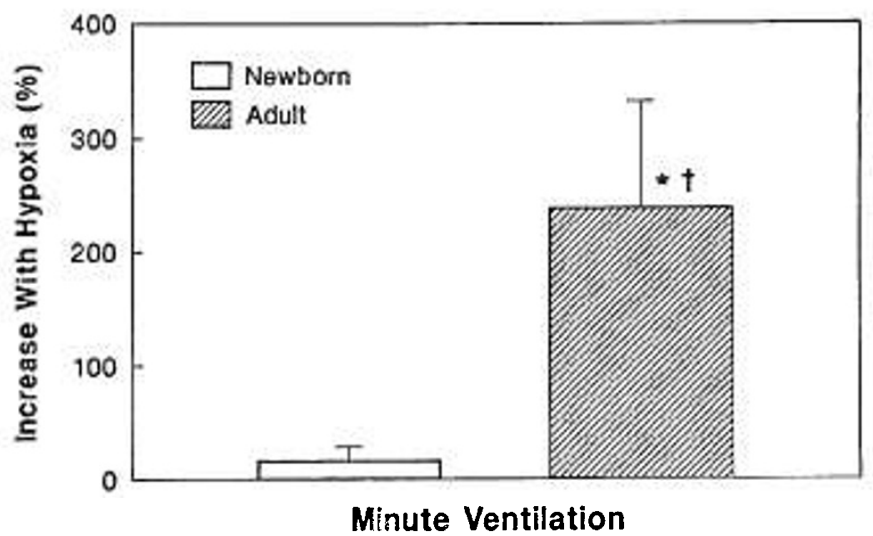

Fig. 4. Ventilatory response to hypoxemia is shown as a percentage of change from the baseline value (protocol 5 ). Values are displayed as means \pm SEM. * , different from the normoxemic baseline $(p<0.05) ; \dagger$, different from the newborn group $(p<0.05)$. 


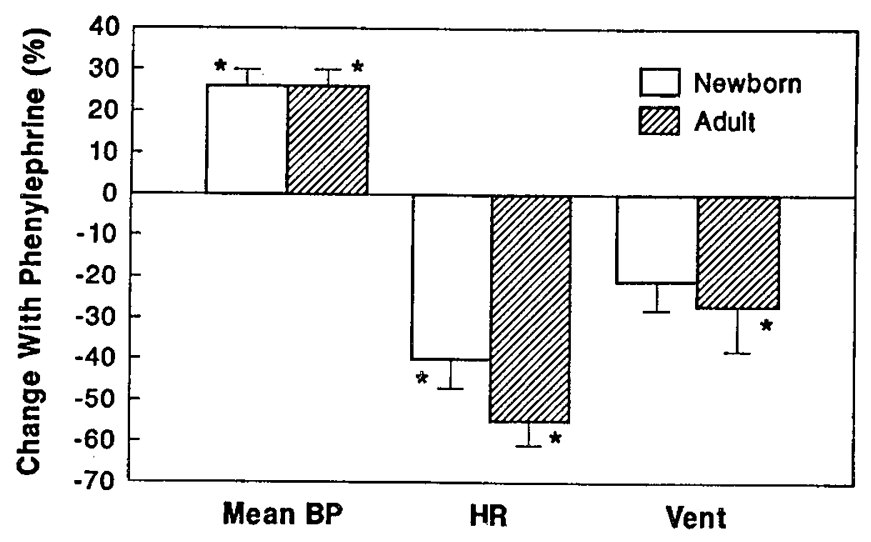

Fig. 5. Blood pressure, heart rate, and ventilatory response to phenylephrine infusion is shown as a percentage of change from the baseline value (protocol 6). Values are displayed as means \pm SEM. $B P$, blood pressure; $H R$, heart rate; Vent, minute ventilation; *, different from the predrug baseline $(p<0.05)$

\section{DISCUSSION}

We evaluated the reflex cardiorespiratory responses to skeletal muscle contraction in newborn and adult cats. We also compared the effects of muscle contraction with the following interventions: 1) direct stimulation of the sciatic nerve afferents, 2) hypoxemia, and 3) phenylephrine infusion. The efferent arm of the exercise pressor reflex is mediated by the sympathetic nervous system, as is the response to sciatic nerve stimulation and to hypoxemia. The efferent limb of the baroreceptor reflex is mediated by the parasympathetic system. We found that newborns had a reduced pressor and heart rate response to muscle contraction compared with the adults, but minute ventilation increased similarly in both groups. There were no differences between the groups regarding stimulation of sciatic afferents or with phenylephrine infusion; hypoxemia was different only in that it stimulated ventilation in the adult much more than in the newborn.

Several mechanisms should be considered as we try to understand our results. Immaturity of this exercise-pressor reflex arc might occur at any one of three levels: 1 ) the afferent limb; 2) the efferent limb; or 3 ) the central integration and processing of the reflex.

Skoglund (16) studied reflexes arising from muscle afferents in the immature kitten. He evaluated proprioceptive and stretch reflexes and found that these mature postnatally because of the development of their afferent links. At birth, peripheral nerves are poorly myelinated, and conduction velocities are consequently quite slow. With time, the nerves become progressively more myelinated, conduction velocities increase, and the simple stretch and proprioceptive reflexes mature (17). However, the increasing conduction velocity in these studies is probably due to myelination of group I and II afferents. Rapid conduction over these afferents is essential for the development of stretch reflexes, but the exercise pressor reflex is mediated by slowly conducting group III and IV afferents. There is little information available about the maturity of these group III and IV muscle afferents at birth.

Downing (18) has recorded nerve activity from A- $\delta$ - and Cfiber afferents from the carotid sinus of the newborn rabbit. He found that, despite low resting blood pressures, the afferents responded to increases in blood pressure in a fashion similar to adult nerves. It is therefore reasonable to speculate that group III and IV muscle afferents (A- $\delta$ and $C$ fibers) function normally at birth, but additional investigations will be needed to test this hypothesis.

Data from the current study supports the idea that the afferent arm of the exercise pressor reflex arc is intact in the newborn. Although the blood pressure and heart rate response to muscle contraction was muted in the newborn, the ventilatory response was virtually identical to that of the adult. This suggests that the afferent limb of the reflex arc is intact, eliciting a normal ventilatory response, but immaturity of the efferent arm or of the central integration of afferent information prohibits a mature pressor and heart rate response. Furthermore, we found no significant age-related differences in the baroreflex, which is also mediated by A- $\delta$ - and C-fiber afferents. This suggests that these small afferent nerves can be mature at birth.

Many studies support the idea that the efferent arm of this pressor reflex could be impaired at birth. Boatman et al. (19) found that sympathetic control of vascular smooth muscle was decreased in the newborn dog. Others have found that adrenergic innervation develops at varying rates in different vascular beds but is generally poor at birth (20). Rockson et al. (21) discovered impaired adrenergic responsiveness in neonatal dog myocardium. In humans, as well as other species, adenylate cyclase activity of the myocardium is decreased at birth and increases with maturity (22). Despite these concerns about the integrity of the efferent limb of the pressor reflex arc, our own data suggest that this does not account for the age-related differences in blood pressure response. Because we observed no age-related differences in the blood pressure response to hypoxemia and sciatic nerve stimulation, we speculate that the muted blood pressure response during muscle contraction is not due to immaturity of the efferent limb of the reflex arc.

Our finding of a muted pressor response to muscle contraction in the newborn is best explained by an immaturity of the CNS. Kalia (23) came to a similar conclusion when she evaluated the $\mathrm{J}$ reflex in newborn kittens. Kalia found a disparity between the rate of maturation of the somatic and the visceral components of this reflex. She felt that this disparity implied an immaturity in the CNS. Pathologic studies have demonstrated a relatively simple axonal architecture in the cortex (24) and spinal gray matter (25) of kittens. In addition, potential central modulators of reflex pressor responses (substance $\mathrm{P}$ and opioid receptors) (26) have been found to be immature at birth and continue to develop postnatally $(27,28)$.

Although chemoreceptor and baroreceptor reflexes seemed similar in newborns and adults, we did note one important difference. Newborns had no significant ventilatory response to hypoxia, whereas adults did increase minute ventilation. This disparity between newborns and adults in response to hypoxia has been recognized and reported in the past $(29,30)$ and probably reflects immaturity of the respiratory control centers.

Methodologic issues should be considered when evaluating our data. The triceps weight in our kittens was significantly less than that of the adults when expressed as a percentage of body weight (Table 1). This suggests that the attenuation of the pressor reflex might have been due to the lesser muscle mass in newborns. However, if fewer afferents were being stimulated in the newborn muscles because of the lesser mass, we might expect an attenuation of the ventilatory component of the reflex as well. Because ventilation was not attenuated in the newborn, we believe that the afferent arm of the reflex was probably similar in the two age groups.

Another methodologic consideration in this model is the intensity of the muscle contraction. The muscle contraction in this model is probably several times longer and more intense than any naturally occurring muscle contraction in the newborn. Because kittens had a muted response to this stimulus, we suspect that spontaneous movements of newborns play little role in the control of blood pressure. We cannot rule out age-related differences in the response to anesthesia or to the surgical preparation; however, we would have expected any differences in response to these portions of the model to impact on all of the reflexes studied.

Generalization of these results to other animals or humans may be inappropriate, because maturation of cardiovascular reflexes has previously been shown to be quite model-specific (31). In general, newborn kittens are probably less mature at 
birth than humans. Kittens have less noradrenaline in the adrenal gland at birth than humans (32). They are also less responsive to dopamine at birth than many other species (33).

In summary, we found that newborn cats have a reduced heart rate and blood pressure response to muscle contraction compared with the adult. We conclude that the exercise pressor reflex plays little or no role in the control of blood pressure in the newborn feline. We speculate that the postnatal development of this reflex is due to maturation of integrative and modulatory mechanisms in the CNS.

\section{REFERENCES}

1. McCloskey DI, Mitchell JH 1972 Reflex cardiovascular and respiratory responses originating in exercising muscle. J Physiol 224:173-186

2. Rotto DM, Stebbins CL, Kaufman MP 1989 Reflex cardiovascular and ventilatory responses to increasing $\mathrm{H}^{+}$activity in cat hindlimb muscle. J Physio 67:256-263

3. Kalia M, Mei SS, Kao FF 1981 Central projections from ergoreceptors (Cfibers) in muscle involved in cardiopulmonary responses to static exercise. Circ Res 48:I48-I62

4. Mitchell JH, Kaufman MP, Iwamoto GA 1983 The exercise pressor reflex: its cardiovascular effects, afferent mechanisms and central pathways. Annu Rev Physiol 45:229-242

5. Mitchell JH 1990 Neural control of the circulation during exercise. Med Sci Sports Exerc 22:141-154

6. Asmussen E, Johansen SH, Jorgensen M, Nielsen M 1965 On the nervous factors controlling respiration and circulation during exercise. Acta Physio Scand 63:343-350

7. Asmussen E, Johansen SH, Jorgensen M, Nielsen M 1963 The neurogenic factors in the regulation of respiration and circulation during muscular exercise. Acta Physiol Scand 59(suppl 213):178(abstr)

8. Lock JE, Einzig S, Moller JH 1978 Hemodynamic responses to exercise in normal children. Am J Cardiol 41:1278-1284

9. Springer C, Barstow TJ, Cooper DM 1989 Effect of hypoxia on ventilatory control during exercise in children and adults. Pediatr Res 25.285-290

10. Kaufman MP, Kozlowski GP, Rybicki KJ 1985 Attenuation of the reflex pressor response to muscular contraction by a substance $\mathrm{P}$ antagonist. Brain Res 377:199-203

11. Kaufman MP, Rybicki KJ, Kozlowski GP, Iwamoto GA 1986 Immunoneutralization of substance $\mathrm{P}$ attenuates the reflex pressor response to muscular contraction. Brain Res 377:199-203

12. Rybicki KJ, Kaufman MP 1985 Stimulation of group III and IV muscle afferents reflexively decreases total pulmonary resistance in dogs. Respi Physiol 59:185-195

13. Turlapaty P, Gootman PM, Altura BT, Alturo BM 1988 Age-related changes in vascular reactivity of neonatal blood vessels. In: Jones CT (ed) Heart and
Cardiovascular Control in Fetal and Neonatal Development. Perinatology Press, New York, pp 145-149

14. Myers JL 1966 Fundamentals of Experimental Design. Allyn and Bacon Boston, pp 174-189

15. Zar JH 1984 Biostatistical Analysis. Prentice-Hall, Englewood Cliffs, NJ, pp 185-189

16. Skoglund S 1960 The spinal transmission of proprioceptive reflexes and the postnatal development of conduction velocity in different hindlimb nerves in the kitten. Acta Physiol Scand 49:318-329

17. Skoglund S, Romero C 1965 Postnatal growth of spinal nerves and roots. Acta Physiol Scand 66(suppl 260):1-50

18. Downing SE 1960 Baroreceptor reflexes in newborn rabbits. J Physiol 150:201-

19. Boatman DL, Shaffer RA, Dixon RL, Brody MJ 1965 Function of vascular smooth muscle and its sympathetic innervation in the newborn dog. $\mathrm{J}$ Clin Invest 44:241-246

20. Duckles SP, Banner W 1984 Changes in vascular smooth muscle reactivity during development. Annu Rev Pharmacol Toxicol 24:65-83

21. Rockson SG, Hamcy CJ, Quinn P, Manders T, Haber E, Vatner SF 1981 Cellular mechanisms of impaired adrenergic responsiveness in neonatal dogs. Clin Invest 67:319-327

22. Vulliemoz J 1984 Developmental changes in adenylate cyclase activity in canine myocardium. Dev Pharmacol Ther 7:409-421

23. Kalia M 1976 Visceral and somatic reflexes produced by $\mathrm{J}$ pulmonary receptors in newborn kittens. J Appl Physiol 41:1-6

24. Gu Q, Patel B, Singer W 1990 The laminar distribution and postnatal development of serotonin-immunoreactive axons in the cat primary visual cortex. Exp Brain Res 81:257-266

25. Windle WF 1930 Normal behavioral reactions of kittens correlated with the postnatal development of nerve-fiber density in the spinal gray matter. Comp Neurol 50:479-503

26. Hill JM, Kaufman MP 1990 Attenuation of reflex pressor and ventilatory responses to static muscular contraction by intrathecal opiods. J Appl Physiol 68:2466-2472

27. Attali B, Soya D, Vogel Z 1990 Pre- and postnatal development of opiate receptor subtypes in rat spinal cord. Dev Brain Res 53:97-102

28. Yew DT, Luo CB, Zheng DR, Guan YL, Lin Q, Chen WZ 1990 Development and localization of enkephalin and substance $P$ in the nucleus of tractus solitarius in the medulla oblongata of human fetuses. Neuroscience $34: 491$ 498

29. De Boeck C, Van Reempts P, Rigatto H, Chernick V 1984 Naloxone reduce decrease in ventilation induced by hypoxia in newborn infants. $\mathbf{J}$ App Physiol 56:1507-1511

30. Gershan WM, Jacobi MS, Thach BT 1990 Maturation of cardiorespiratory interactions in spontaneous recovery from hypoxic apnea. Pediatr Res 28:8793

31. Gootman PM, Gootman N, Buckley BJ 1983 Maturation of central autonomic control of the circulation. Fed Proc 42:1648-1655

32. Comline RS, Silver M 1966 Development of activity in the adrenal medulla of the foetus and newborn animal. Br Med Bull 22:16-20

33. Buckley NM 1986 Maturation of circulatory system in three mammalian models of human development. Comp Biochem Physiol 83A:1-56 